\title{
Research Progress of Nrf2 Signaling Pathway on Diabetic Nephropathy
}

\author{
Jingbo Li \\ The Second Affiliated Hospital of Qiqihaer Medical University, Heilongjiang Qiqihaer 61006, China
}

Keywords: Nrf2 signaling pathway; diabetic nephropathy; advances

\begin{abstract}
The transcription factor NF-EZ-related factor 2 (NF-EZ-erlated factor Z, N-argument) is an important transcription factor that regulates oxidative stress in vivo and is a central regulator of cellular anti-oxidation and repression. $\mathrm{N}$-curd is activated by interaction with antioxidant response elements. The Nrf2 A/RE pathway, which reduces reactive oxygen species produced by high-glucose factors, exerts its anti-oxidative stress-damaging and anti-chronic inflammatory responses, and improves oxidative stress-induced insulin resistance, and a defense mechanism plays a very important role in diabetic macrovascular and microvascular complications.
\end{abstract}

\section{Introduction}

Diabetes melliuts (DM) is a chronic metabolic disorder of abnormal glucose metabolism and insulin resistance. Sustained hyperglycemia produces excessive reactive oxygen species, resulting in decreased antioxidant enzyme activity, induction of insulin resistance, and pathways through the end-glycosylation end products (AGES) pathway, the polyol pathway, the protein kinase C activation pathway, the hexosamine pathway, etc. A variety of mechanisms lead to chronic inflammatory response and oxidative stress injury in the cardiovascular system. Major vascular and microvascular complications are the major causes of disability and death in diabetic patients. Nuclear factor 2 related factor 2 deletion or activation disorder, NA / RE pathway is inactive, which exacerbates the oxidative stress of the cytotoxicity, endothelial dysfunction, accelerated macrovascular, microvascular disease The occurrence and development. Activation of $\mathrm{Nl} \mathrm{A/RE}$ pathway reduces reactive oxygen species production, improves oxidative stress-induced insulin resistance, and corrects hyperglycemia-induced tissue biochemical dysfunction leading to its anti-oxidative stress and cytoprotection against chronic inflammatory responses The function plays a very important role in the defense mechanism of diabetic macrovascular and microvascular complications.

\section{Nrf2 Signaling Pathway}

Nrf2 is a nuclear transcription factor in the basic leucine zipper family and has a molecular weight of about $66 \mathrm{kDa}$. Moi et al. (1994) found that Nrf2 binds to the NF-E2 repeat of the B-globin gene promoter and can be cloned and Highly expressed in the kidney. Nrf2 can promote the detoxification and excretion of toxic and harmful substances, increase antioxidant activity and anti-inflammatory effects, and improve mitochondrial function. It can also effectively remove dysfunctional organelles and aggregates of toxic proteins by enhancing autophagy [1]. Under normal conditions, Nrf2 and cytoskeleton-related proteins exist in the cytoplasm in the form of dimers, when the body ROS* fund project: Zunyi City Science and Technology Project [No: Zunshikesheshe No.; Master Affiliated hospital of Zunyi Medical College research project started No: Institute word No. 1 Graduate School of Medical College, Zunyi, Guizhou Province Affiliated hospital of Zunyi Medical emergency department of communication: Li Jianguo, E -mail:Lijianguo78211@sina. When comgenesis and clearance of com are unbalanced, the body itself can induce a series of protective proteins in order to relieve the damage to cells. This coordinated response is regulated by the AREs in the upstream regulatory regions of these protective genes. Recent studies have found that Nrf2 is an ARE activation factor that binds to Maf, etc., and then binds to the ARE promoter to initiate the expression of a variety of downstream 
protective genes. These downstream genes include heme oxygenase-1 (HO -1) Catalase (CAT), Superoxide Dismutase (SOD), Glutathione Sulfur Transferase (GST), etc., to improve the anti-oxidation, anti-tumor, anti-inflammatory and anti-witherance of cells and tissues. Ability to die. At present, Nrf2 signaling pathway has been studied in the lung diseases, liver diseases, nervous system diseases and other areas, and kidney disease has gradually begun to study. The Nrf2 pathway has gradually become a new target for the treatment of renal system diseases.

\section{Nrf2 Signal Pathway and Kidney Disease}

Diabetic nephropathy (DN) is the leading cause of end-stage renal failure and is one of the most common chronic microvascular complications of diabetes. The occurrence of DN is associated with hyperglycemia, hypertension, hyperlipidemia, obesity, inflammation, and oxidative stress. Currently, oxidative stress and inflammation have been confirmed to play an important role in the pathogenesis of DN. Studies have shown that macrophages infiltrate different stages of DN kidney inflammatory cells and produce ROS, pro-inflammatory cytokines and growth factors, etc. The main inflammatory cytokines involved in the development of DN include IL-6, IL-1, IL-18 and TNF- $\alpha$ [2]. Diabetic patients can produce large amounts of ROS. Kidneys are organs that are sensitive to ROS damage. When ROS is abundantly present in renal parenchymal cells, it reduces or even destroys the ability of the kidneys to scavenge free radicals, thereby increasing the synthesis of extracellular matrix proteins, glomeruli. , The tubular basement membrane and mesangial matrix increased, eventually glomerulosclerosis and tubulointerstitial fibrosis, and then developed into DN. It has been reported that Nrf2 expression is elevated in diabetic nephropathy, and ROS production is increased after knocking out the Nrf2 gene, and oxidation causes DNA damage and kidney damage. Activation of Nrf2 has now been confirmed as a potential target for the treatment of diabetes and can prevent kidney oxidation. Stress and chronic inflammation can also negatively regulate transforming growth factor-B, improve metabolic disorders, and further reduce diabetic kidney damage. Experimental studies have found that fenofibrate can significantly increase the expression of fibroblast growth factor 21 (FGF21) by activating the Nrf2 pathway to protect kidney damage caused by diabetes. Naringin has also been shown to improve renal function by inhibiting oxidative stress by activating Nrf2, inhibiting collagen formation and renal interstitial fibrosis. In addition, hyperbaric oxygen therapy (HBOT) has also been found to activate the antioxidant defense system of Nrf2 and cells to reduce diabetic kidney injury. At present, different experiments have also found that tert-butyl hydroquinone, asiatic acid, grape seed proanthocyanidins, sulforaphane and sulforaphane and cinnamaldehyde can be used as Nrf2 inducers to upregulate Nrf2 levels, or through direct injection of Nrf2. The expression plasmid initiates the Nrf2 signaling pathway to reduce renal injury.

Acute kidney injury is very common in clinical practice, and the mortality is high (24\% to 26\%), especially after kidney transplantation, partial resection of the kidneys, and heart surgery. Antioxidation is currently found to be an effective strategy for the treatment of acute kidney injury (AKI). Zhang et al. (9) found that mesenchymal stem cell extracellular vesicles (MSCEVS) can activate the Nrf2 pathway, up-regulate HO-1 in vitro and in vivo, and reduce hypoxia-induced damage in renal tissue and tubular epithelial cells. Liu et al. (10) found that the incidence of acute kidney injury caused by iohexol can be reduced by the action of phosphorylated glycogen synthase kinase- $3 \beta$ and the inhibition of NF- $\mathrm{kB}$ by activation of Nrf2 to reduce oxidative stress and inflammatory responses. Intermittent hypoxia-induced renal injury is the main clinical feature of obstructive sleep apnea syndrome (OSA). Wu et al. [3] concluded through an experiment that metallothionein prevents intermittent hypoxia-induced renal injury in Nrf2. Signaling plays a key role. Zahran et al. (12) found that sildenafil can activate antioxidant anti-apoptotic genes and inhibit pro-inflammatory cytokine genes in renal ischemia-reperfusion injury in rats, further protecting the kidneys, including the Nrf2 pathway. Plays an important role. Propofol preconditioning can reduce autologous orthotopic liver transplantation to induce distal renal injury, suggesting that Nrf2 plays an important role in protecting oxidatively injured cellular responses. Cisplatin is a chemical agent used in the treatment of tumors. Clinical use often leads to varying degrees of nephrotoxicity and 
even to acute renal failure. Nrf2 can be used as a transcription factor in renal protection [5]. It was found that baicalein pretreatment can reduce renal oxidative stress, apoptosis, and inflammatory reaction induced by cisplatin, further improve renal injury and restore renal function. At the same time, it was found that the levels of Nrf2 and downstream HO-1 in the kidneys were elevated, and thus confirmed. The Nrf2 pathway plays a role in cisplatin-induced acute renal failure.

Chronic kidney disease (CKD) is a complex disease with a high incidence and no single treatment at present. Since the discovery of a significant increase in biological markers representing oxidative stress in CKD patients, oxidative stress has attracted attention as a pathological factor of chronic kidney disease. The Nrf2 pathway is also increasingly recognized as a powerful antioxidant stress pathway. Numerous studies have demonstrated that Nrf2 activators play a protective role in chronic kidney disease and curcumin is used as a dietary supplement in diabetic or non-diabetic kidney injury patients. Both can activate the Nrf2 pathway to reduce oxidative stress and protect the kidney. Studies have also shown that both biologically active compounds and physical exercise can lead to the expression of Nrf2, and found that physical exercise can be used as a non-pharmaceutical method to benefit patients with CKD by activating the Nrf2 pathway. Nrf2 is a regulator of anti-oxidation and detoxification enzyme genes and related proteins. The data of expression of Nrf2 and its key target gene products in CKD patients is limited, and the expression of NF- $\mathrm{kB}$ in peripheral blood mononuclear cells and Nrf2, NQO1 in hemodialysis patients. The expression was negatively correlated. These observations suggest that the Nrf2 pathway may reduce oxidative stress and inflammatory responses. Necrotic crescentic glomerulonephritis (RPGN) is a life-threatening syndrome characterized by rapid loss of kidney function. There is evidence that the podocyte-expressed transcription factor peroxisome proliferator-activated receptor (PPAR $\gamma$ ) protects against podocyte injury, but Nrf2-deficient mouse glomerular podocytes also lose PPAR $\gamma$, establishing Nrf2-PPAR $\gamma$ podocytes Cascade, these transcription factors can enhance glomerular tolerance, to avoid the damage mediated by severe immune complexes, Nrf2-PPAR $\gamma$ pathway may be a new target for RPGN treatment.

Renal interstitial fibrosis is thought to be an end-stage renal disease due to oxidative damage. Angiotensin II can cause kidney fibrosis, and by establishing a mouse model of unilateral ureteral obstruction induced renal fibrosis (UUO), the application of angiotensin II After treatment with receptor antagonists, changes in the renal renin-angiotensin system (RAS) in the obstructive side were evaluated, as well as expression of Nrf2 and its downstream antioxidant genes, as well as renal inflammation, fibrosis, and apoptosis, demonstrating vascular tone. Receptor II antagonists reduce renal oxidative stress, inflammation, and fibrosis by affecting RAS and MAPK and Nrf2 signaling pathways.

\section{Conclusion}

The Nrf2 signaling pathway has become a research hotspot in many diseases of the kidney. Nrf2, as a key factor in inhibiting oxidative stress, is involved in the pathogenesis and development of renal diseases. Numerous experiments have confirmed that Nrf2 signaling pathway has a protective effect on renal system diseases. Studies on autoimmune-associated nephropathy and renal damage caused by acute poisoning are still controversial, and a large number of clinical trials are needed to further confirm the study.

\section{References}

[1] Xiao Dongwei, Gao Mingli .Discussion on theoretical basis of acupoint application [ J ] .Liaoning Traditional Chinese Medicine University Journal, 2008, 10 (5): 127.

[2] Zhang Yanhong. Theoretical basis of acupoint application and current application status [J]. Traditional Chinese Medicine, 2007, 20 (2): 1-3.

[3] Liu Weiai, Ye Debao .Research progress of transdermal administration of acupoint application therapy [J]. Medical Bulletin, 2004, 3 (4):59-62. 
[4] Qian Yuping. White mustard seeds scattered topical treatment of children with pneumonic rales malabsorption in 84 cases [J]. Journal of Henan University (Medical Science), 2006, 25 (1): 60-61.

[5] Wang Jingbo, Wang Zuozhong, Wei Lanfang. Combination of Chinese and Western medicine for internal and external treatment of children with pneumonia and asthma Observation of 60 cases [J]. New Chinese Medicine, 2010 (11): 33-34. 